\title{
Malformaciones vasculares: Manejo mediante técnicas de radiología intervencional y satisfacción de los pacientes
}

Drs. Patricio Vargas $H^{(1)}$, Benjamín Horwitz $Z^{(2)}$, TM. Cecilia Fábrega $G^{(3)}, T M$. Nitza Espinosa $R^{(3)}$, Drs. Claudio Silva $F^{(1)}$, Juan Pablo Niedmann $E^{(1)}$, Víctor Bianchi ${ }^{(4)}$, Carlos Giugliano $V^{(5)}$, Rodrigo Raurich $\mathbf{S}^{(2)}$.

1. Radiólogo, 2. Becado de Radiología, 3. Tecnólogo Médico, 4. Cirujano Vascular Periférico, 5. Cirujano Plástico.

Facultad de Medicina, Clínica Alemana de Santiago. Universidad del Desarrollo. Santiago, Chile.

Vascular malformations: Interventional radiology management and assesment of patient satisfaction

Abstract: Background: Vascular anomalies are prevalent in the general population and may produce significant impairment in quality of life. In recent years, minimally invasive interventional radiology techniques have become an efficient alternative for the treatment of these patients. The aim of this study was to present our experience in the management of vascular malformations with such techniques at Clínica Alemana in Santiago, Chile, and assess the satisfaction of patients with this management.

Material and Methods: Evaluation of the follow-up of patients with vascular malformations treated in the Interventional Radiology Unit at our center since June - 2006 to June - 2009. Patients were classified according to the hemodynamics of the lesions (high vs low-flow). Complications and patient satisfaction with the treatment were assessed.

Results: Eighty-seven patients (253 procedures) were included in the study, 36 men and 51 women, all with complete follow-up during the period of the study. Their average age was 20.6 years. In over $50 \%$ of the patients the vascular anomaly was located on the face or the lower extremities. Twenty-eight percent (25 patients) had a high-flow malformation (pure or mixed) and 62 (72\%) had a low-flow lesion (vascular malformation, lymphangioma or mixed). On average, each patient underwent 2.9 procedures (1-10). There were 4 major complications (1.65\%), one of which was predominantly cosmetic with necrosis in the area of the lesion. The average satisfaction rate was 7.95 (on a rating scale of 1 to 10). Satisfaction was significantly higher in patients with low-flow malformations (8.27) than in those with high-flow lesions $(7.16)$. $(p<0.05)$ Discussion: Treatment of vascular malformations using interventional radiology techniques is an effective alternative, with good prospects of clinical improvement, patient satisfaction and low complication rates. Keywords: Interventional radiology techniques, Patient satisfaction, Vascular anomalies.

Resumen: Introducción: Las anomalías vasculares son una patología frecuente en la población general, y son causa de deterioro de la calidad de vida de los pacientes que las padecen. En los últimos años, las técnicas mínimamente invasivas de radiología intervencional se han convertido en una alternativa eficaz para el tratamiento de estos pacientes. El objetivo del presente estudio es mostrar nuestra experiencia en el manejo de malformaciones vasculares con estas técnicas en la Clínica Alemana de Santiago y evaluar la satisfacción de los pacientes con este manejo.

Material y Métodos: Evaluación del seguimiento de todos los pacientes con malformaciones vasculares tratados en la Unidad de Radiología Intervencional desde el año 2006 hasta junio del año 2009. Los pacientes fueron clasificados en base a la hemodinamia de la malformación vascular (alto flujo vs. bajo flujo). Se evaluaron las complicaciones, y se realizó una encuesta sobre la satisfacción de los pacientes con el tratamiento.

Resultados: Ochenta y siete pacientes (253 procedimientos) fueron incluidos en el estudio, 36 hombres y 51 mujeres, todos con seguimiento completo en este periodo. La edad promedio de ellos fue de 20,6 años. En más del $50 \%$ de los pacientes la anomalía vascular se ubicó en la cara o extremidades inferiores. Un 28\% (25 pacientes) presentó malformación de alto flujo (pura o mixta) y 72\% (62 pacientes) de bajo flujo (malformaciones vasculares, linfangiomas o mixtas). En promedio, cada paciente se realizó 2,9 procedi- 
mientos (1-10). Del total de procedimientos realizados, hubo 4 complicaciones mayores (1,65\%), una de ellas con secuelas estéticas producto de necrosis de la zona. El promedio de satisfacción fue de un 7,95 en una escala de 1 a 10, siendo significativamente mayor en pacientes con malformaciones de bajo flujo $(8,27)$ que en los de alto flujo $(7,16)(p<0.05)$.

Discusión: El tratamiento de las malformaciones vasculares mediante técnicas de radiología intervencional es una alternativa eficaz, con buenas expectativas de mejoría clínica, satisfacción de los pacientes y baja tasa de complicaciones.

Palabras clave: Anomalías vasculares, Satisfacción del paciente,Técnicas de radiología intervencional.

Vargas $P$. Malformaciones vasculares: Manejo mediante técnicas de radiología intervencional y satisfacción de los pacientes. Rev Chil Radiol 2011; 17(2): 85-89.

Correspondencia a: Dr. Patricio Vargas H. / pvargas@alemana.cl

Trabajo premiado en categoría oral, durante el XXV Congreso Interamericano de Radiología, efectuado en Santiago de Chile.

\section{Introducción}

Las anomalías vasculares son una patología frecuente en la población general, y pueden provocar alteraciones cosméticas, infecciones recurrentes, alteraciones funcionales, falla cardíaca, hemorragias, entre otras; $y$ son causa de deterioro de la calidad de vida de los pacientes que las padecen ${ }^{(1)}$. Su clasificación general las divide en tumores (hemangiomas) y malformaciones vasculares.

La clasificación de las anomalías vasculares ha sido objeto de múltiples variaciones en los últimos años, con el objeto de clarificar que los hemangiomas y las malformaciones vasculares son patologías diferentes y su tratamiento es distinto.

Jackson y colaboradores propusieron en 1993 dividir las malformaciones vasculares en base a su hemodinamia(2), convirtiéndose en la clasificación más utilizada en la actualidad.

Existen varias alternativas para el tratamiento de estas patologías, las que incluyen el manejo conservador médico-dermatológico, quirúrgico y la radiología intervencional. Esta última ha tenido un gran avance en los últimos años a través del desarrollo de nuevos agentes embolizantes y la aplicación de técnicas mínimamente invasivas ${ }^{(1)}$ (Figura 1). El manejo mediante embolización transarterial es generalmente considerado la mejor alternativa para el tratamiento de las malformaciones de alto flujo. Para las de bajo flujo, la punción percutánea directa con agentes embolizantes ha tenido buenos resultados ${ }^{(3-5)}$.

Existen pocos reportes en la literatura internacional acerca de los resultados a corto y largo plazo del tratamiento mediante radiología intervencional de estas patologías ${ }^{(4-7)}$ y la mayoría de ellos incluyen la experiencia local con series pequeñas. En Chile hay escasa experiencia reportada, destacando un estudio sobre manejo quirúrgico de hemangiomas en niños ${ }^{(8)}$, y otro que revisa la patología desde el punto de vista del diagnóstico por imágenes ${ }^{(9)}$. No hay trabajos publicados a la fecha acerca de los resultados a largo plazo en el manejo de malformaciones vasculares periféricas mediante radiología intervencional.
El objetivo del presente estudio es mostrar la experiencia en el manejo de malformaciones vasculares con técnicas de radiología intervencional en la Clínica Alemana de Santiago. Se evaluaron los resultados a corto y mediano plazo, se analizaron las complicaciones, y por último, se valoró el grado de satisfacción de los pacientes con la consecuente repercusión en su calidad de vida.

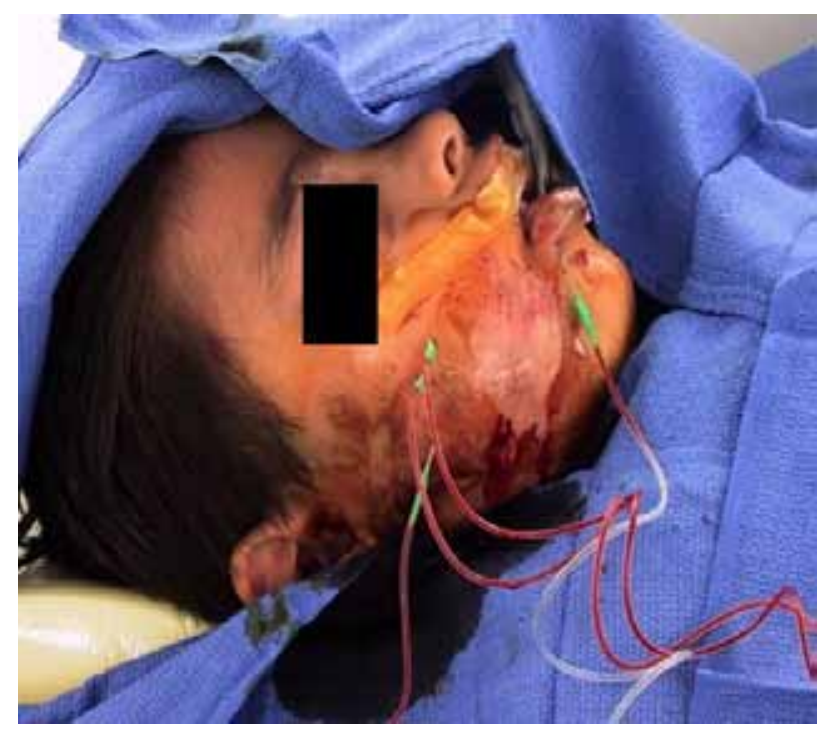

Figura 1. Paciente con malformación de bajo flujo en la mejilla durante procedimiento de embolización percutánea con Polidocanol.

\section{Material y métodos}

Se llevó registro de pacientes con diagnóstico de malformaciones vasculares sometidos a tratamiento con técnicas de radiología intervencionista en la Clínica Alemana de Santiago, se incluyeron todos los pacientes tratados en la Unidad de Radiología Intervencional desde el año 2006 hasta junio del año 2009. La decisión sobre el tipo de manejo que recibió cada uno de los pacientes fue definida en una reunión mensual de anomalías vasculares por 
un equipo multidisciplinario constituido (entre otros) por cirujanos, dermatólogos, médicos internistas y radiólogos intervencionistas.

Los pacientes fueron clasificados en base a la hemodinamia de malformación vascular según los criterios de Jackson ${ }^{(2)}$ y la clasificación acordada por la Sociedad Internacional para el Estudio de Anomalías Vasculares ISSVA ${ }^{(10)}$ (linfática, vascular de bajo flujo, vascular de alto flujo, mixtas linfática y vascular de bajo flujo, mixtas linfática y vascular de alto flujo).

Las complicaciones fueron clasificadas de acuerdo a las guías de la Sociedad de Radiología Intervencional de Estados Unidos ${ }^{(11)}$ en tipo A, complicación menor que se resuelve en forma espontánea y no requiere tratamiento; tipo $\mathrm{B}$, complicación menor que requiere tratamiento conservador; tipo $\mathrm{C}$, complicación mayor que requiere tratamiento que incluye hospitalización por menos de $48 \mathrm{hrs}$; tipo $\mathrm{D}$, complicación que requiere tratamientos mayores (que requiere hospitalización de mayor cuidado y por más de 48 hrs.); tipo E, complicación mayor con secuelas permanentes; y tipo F, con resultado de muerte del paciente.

Se construyó una base de datos utilizando el software MS Excel 2003 $\AA$ (Microsoft Corporation $\AA$ ), y se recolectaron los siguientes datos: edad al momento del diagnóstico, fecha del primer procedimiento, tratamientos previos a los que fue sometido, número y tipo de intervenciones realizadas, agentes embolizantes empleados, complicaciones tempranas y tardías.

Finalmente, se les consultó a los pacientes su grado de satisfacción con el tratamiento pidiéndole que lo califiquen en una escala de $1 \mathrm{a} 10$, siendo un resultado malo, 5 resolución parcial pero insuficiente, y 10 resolución completa de los síntomas. Se les solicitó que consideren el grado de resolución de los síntomas que motivaron su consulta, y la mejoría estética. Se compararon los resultados en el grado de satisfacción de pacientes con malformaciones vasculares de alto flujo versus bajo flujo, utilizando el test de Wilcoxon-Mann-Whitney considerando un $\mathrm{p}<0.05$ como estadísticamente significativo. De la misma manera se compararon los resultados entre aquellos pacientes que completaron todas las sesiones de tratamiento con los que aún no han terminado.

\section{Resultados}

Ochenta y siete pacientes fueron incluidos en el estudio, 36 hombres y 51 mujeres (Tabla I). La edad promedio de ellos fue de 20,6 años ${ }^{(1-59)}$. Al momento del análisis de los resultados, 63 pacientes estaban de alta y no requerían más sesiones de tratamiento mínimamente invasivo. Los restantes 24 pacientes estaban aún en control, con sesiones de tratamiento pendientes por realizarse.

Veinticuatro pacientes $(27,6 \%)$ habían recibido tratamientos previos a la realización de su primera sesión de terapia con radiología intervencional, los cuales consistieron en cirugías ${ }^{(22)}$, biopsias incisionales o escisionales ${ }^{(9)}$, terapia con OK-432 Picinabil liofilizado ${ }^{(1)}$, uso de nieve carbónica ${ }^{(1)}$, y/o esclerosis con bleomicina ${ }^{(1)}$.

Tabla I. Características de los Pacientes.

\begin{tabular}{lc} 
Edad & $\begin{array}{c}\mathbf{2 0 , 6} \mathbf{1 2 , 9} \\
(\mathbf{1}-\mathbf{5 9}) \text { años }\end{array}$ \\
\hline $\begin{array}{l}\text { Sexo } \\
\text { Hombres }\end{array}$ & $36(41,4 \%)$ \\
Mujeres & $51(58,6 \%)$ \\
Pacientes aún en control & $24(27,6 \%)$ \\
Alto flujo & $10(11,5 \%)$ \\
Bajo flujo & $14(16,1 \%)$ \\
Pacientes de alta & $63(72,4 \%)$ \\
Alto flujo & $15(17,2 \%)$ \\
Bajo flujo & $48(55,2 \%)$ \\
\hline
\end{tabular}

En más de la mitad de los pacientes la anomalía vascular se ubicó en la cara o extremidades inferiores (Tabla II). De los 87 pacientes, 25 (28\%) presentaron malformación de alto flujo (puras y mixtas), y 62 (72\%) de bajo flujo (malformaciones vasculares, linfangiomas, o mixtas). El detalle se ilustra en la Tabla III (Figuras. 2 y 3 ).

Tabla II. Ubicación de malformaciones vasculares.

\begin{tabular}{|lrr|} 
Ubicación & $\mathbf{N}^{\circ}$ de & Pacientes \\
\hline Cabeza y cuello & 36 & $(41,4 \%)$ \\
Extremidades Inferiores & 32 & $(36,8 \%)$ \\
Región pelviana & 6 & $(6,9 \%)$ \\
Cuello & 5 & $(5,7 \%)$ \\
Extremidades superiores & 4 & $(4,6 \%)$ \\
Tórax & 3 & $(3,4 \%)$ \\
Lumbar & 1 & $(1,1 \%)$ \\
\hline
\end{tabular}

Tabla III. Tipos de malformaciones vasculares.

Bajo flujo $62(71,3 \%)$

$\begin{array}{lrr}\text { Bajo flujo } & 51 & (58,6) \\ \text { Linfangioma } & 7 & (8,0 \%) \\ \text { Linfangioma + bajo flujo } & 3 & (3,4 \%) \\ \text { FAV de bajo flujo } & 1 & (1,1 \%)\end{array}$

Alto flujo $25(28,7 \%)$

Alto flujo 23

Linfangioma + alto flujo

2 


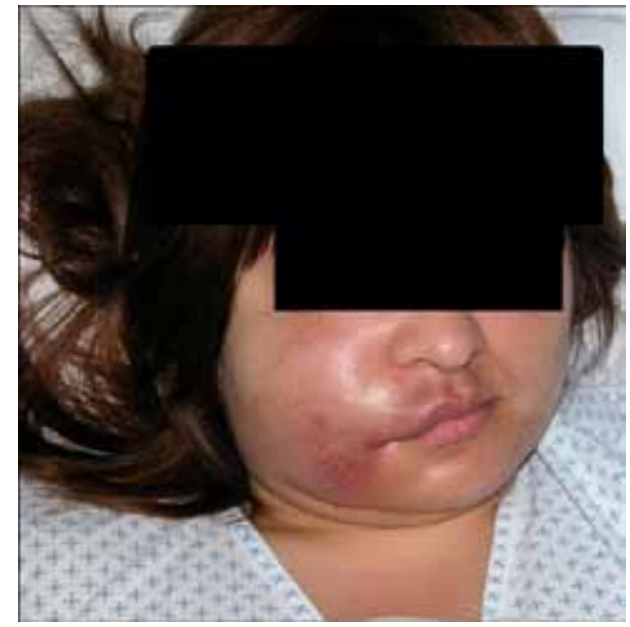

Figura 2. Paciente con malformación de alto flujo antes del tratamiento y después del tratamiento.

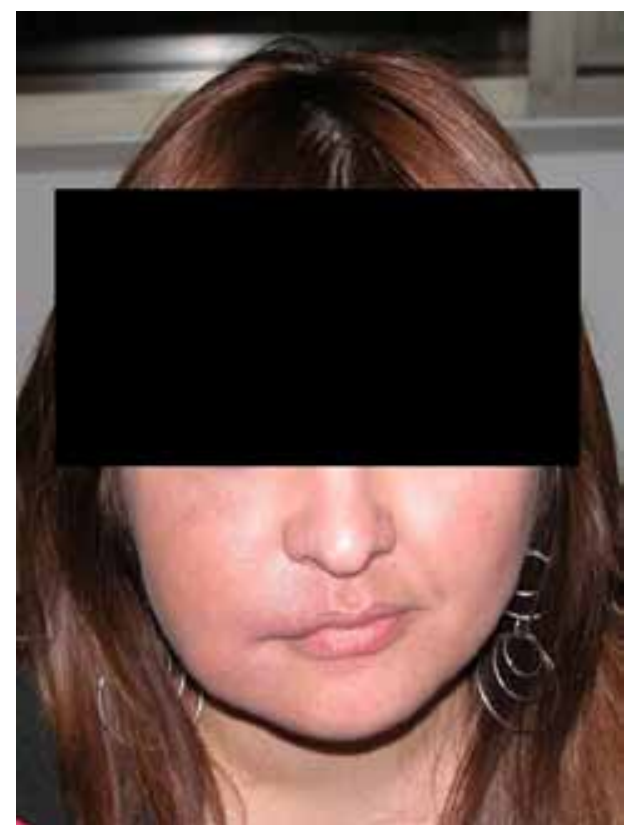

Figura 3. Nótese importante reducción de volumen y mejoría estética.

Dentro de los motivos de consulta, un $89 \%$ lo hizo por aumento de volumen, $72 \%$ de los pacientes consultó por dolor, $54 \%$ por motivos cosméticos, y $37 \%$ por presentar algún tipo de deterioro funcional.

Hasta junio del año 2009, 34 pacientes habían sido sometidos a una sola sesión (39,1\%), 17 pacientes a dos sesiones (19,1\%), 11 pacientes a tres sesiones (12,6\%), 6 pacientes a cuatro sesiones $(6,9 \%)$, y 7 a pacientes cinco sesiones (8\%). Los restantes pacientes se realizaron entre 6 y 10 sesiones. El intervalo promedio entre sesiones fue de 60 días $^{(49-93)}$. En total, se efectuaron 253 procedimientos. Ocho pacientes fueron intervenidos quirúrgicamente como manejo final (9,2\%): Dos de los 62 pacientes con lesión de bajo flujo $(3,2 \%)$ y 6 de los 25 pacientes con lesión de alto flujo (24\%).
En 78 pacientes $(89,7 \%)$ se utilizó polidocanol como agente embolizante. De ellos, en 17 casos $(21,8 \%)$ casos se utilizó en combinación con otros compuestos (alcohol, lipiodol, cianoacrilato, histoacryl). En 9 pacientes se utilizaron combinaciones de algunos de estos últimos agentes. El volumen promedio de polidocanol utilizado en cada sesión fue de $4,3 \mathrm{cc}(2-10 \mathrm{cc})$.

Del total de procedimientos realizados, hubo 4 complicaciones mayores (1,65\%): 1 tipo $\mathrm{E}$ según las guías de la Sociedad de Radiología Intervencional y 3 tipo C. El paciente con la complicación más grave presentó necrosis de la región facial que requirió cirugías reconstructivas y quedó con secuelas estéticas. De los pacientes con complicaciones tipo $\mathrm{C}$, uno presentó neuropatía del primer ortejo del pie izquierdo, que se manejó con analgesia y Gabapentina, logrando control de la sintomatología después de 3 meses de tratamiento. Otro paciente presentó necrosis superficial de la piel de la región lumbar izquierda, que se manejó con curaciones y analgesia, obteniendo buenos resultados con resolución completa a los 3 meses. Un último paciente presentó trombosis de la vena poplítea, al cabo de tres meses de tratamiento con anticoagulación los síntomas cedieron. Ninguno de ellos requirió hospitalización.

En cuanto a la satisfacción de los pacientes, el promedio de satisfacción fue de un 7,95 en una escala de 1 a 10 (Tabla IV). La satisfacción de los pacientes que tenían malformación de bajo flujo fue mejor que la de los pacientes con malformación de alto flujo ( 8,27 vs. 7,16$)$, y también fue mayor en los pacientes que estaban de alta respecto de los que aún tenían sesiones pendientes de tratamiento $(8,14$ vs 7,46$)$. Ambas diferencias fueron estadísticamente significativas con un $p<0.05$ aplicando el test de Wilcoxon-Mann-Whitney.

Tabla IV. Satisfacción de los Pacientes con el Tratamiento.

Tipo de malformación Promedios, DS y rango

\begin{tabular}{|c|c|c|}
\hline $\begin{array}{l}\text { Bajo flujo } \\
\text { Aún en control } \\
\text { de alta }\end{array}$ & $\begin{array}{l}8,27 \pm 0,89 \\
8,00 \pm 0,68 \\
8,35 \pm 0,93\end{array}$ & $\begin{array}{l}(7-10) \\
(8-10) \\
(7-10)\end{array}$ \\
\hline $\begin{array}{l}\text { Alto flujo } \\
\text { Aún en control } \\
\text { de alta }\end{array}$ & $\begin{array}{l}7,16 \pm 1,52 \\
6,70 \pm 1,16 \\
7,47 \pm 1,68\end{array}$ & $\begin{array}{r}(5-10) \\
(5-9) \\
(6-10)\end{array}$ \\
\hline Total & $7,95 \pm 1,21$ & $(5-10)$ \\
\hline Aún en control & $7,46 \pm 1,10$ & $(6-9)$ \\
\hline De alta & $8,14 \pm 1,20$ & $(6-10)$ \\
\hline
\end{tabular}




\section{Discusión}

Las malformaciones vasculares son una patología de difícil tratamiento y provocan importante deterioro en la calidad de vida de los pacientes. En este estudio exponemos nuestra experiencia en el manejo percutáneo durante los últimos 4 años. Nuestros pacientes han presentado hasta la fecha importante mejoría de su sintomatología. En los 242 procedimientos realizados hasta junio del año 2009 , sólo $4(1,5 \%)$ presentó complicaciones, una de las cuales fue grave dejando secuelas estéticas y las otras 3 fueron moderadas, con resolución completa de los síntomas en un plazo no mayor a 3 meses.

El 9,2\% de los pacientes incluidos en nuestro estudio fue sometido a cirugía como manejo final. Las cirugías son consideradas como procedimientos de mayor seguridad debido a su capacidad de lograr un buen control hemostático durante el procedimiento. Los pacientes con lesiones de alto flujo son los que más requirieron cirugía y la mayoría de los pacientes con lesiones de bajo flujo no requirieron cirugía. Por todo lo anterior, creemos que la cirugía ocupa un rol fundamental dentro del manejo de estos pacientes.

Nuestros resultados demuestran que esta terapia logra una adecuada aceptación y satisfacción en los pacientes que la reciben. Cabe destacar que estas patologías pueden ser causa de importante deterioro en la calidad de vida de los pacientes, tanto por la sintomatología física como por el trasfondo psicológico a la que conducen. Este último factor podría explicar la menor satisfacción de los pacientes con malformaciones vasculares de alto flujo, ya que por lo general en ellos el tratamiento es más dificultoso y conllevan a un mayor deterioro de la autoestima y a frustraciones acumuladas que en los pacientes con malformaciones de bajo flujo. Estas nuevas técnicas podrían marcar una diferencia respecto de las utilizadas anteriormente.

Nuestros resultados concuerdan con los reportados previamente en la literatura ${ }^{(7,12,13)}$ donde se reportan tasas de satisfacción de entre un $58 \%$ y un $100 \%$.

\section{Bibliografía}

1. Legiehn G, Heran M. Classification, diagnosis, and interventional radiologic management of vascular malformations. Orthop Clin North Am 2006; 37(3): 435-474.

2. Hyodoh H, Hori M, Akiba H, Tamawaka M, Hydoh K, Hareyama M. Peripheral vascular malformations: imaging, treatment approaches, and therapeutic issues. Radiographics 2005; 25(Suppl 1): S159-171.

3. White R, Pollak J, Persing J, Henderson K, Thomson $J$, Burdge G. Long-term outcome of embolotherapy and surgery for high-flow extremity arteriovenous malformations. JVIR 2000; 11(10): 1285-1295.

4. Do $Y$, Yakes W, Shin W. Ethanol embolization of arteriovenous malformations: interim results. Radiology 2005; 235(2): 674-682.

5. Lee B, Kim D, Huh S. New experiences with absolute etanol sclerotherapy in the Management of a complex formo f congenital venous malformations. J Vasc Surg 2001; 33: 764-772.

6. Donnelly L, Adams D, Bisset G. Vascular malformations and hemangiomas: a practical approach in a multidisciplinary clinic. AJR 2000; 174: 1285-1295.

7. Van der Linder $E$, Pattinama $P$, Heeres $E$, de Jong $S$, Hop W, Kroft L. Long-term patient satisfaction after percutaneous treatment of peripheral vascular malformations. Radiology 2009; 251(3): 926-932.

8. Morovic $C$, Vidal $C$, Acevedo $C$. Tratamiento quirúrgico de hemangiomas en niños. Rev Chil Pediatr 2000; 71(1): 17-23.

9. Arce J, García C, Otero J, Villanueva E. Anomalías vasculares de partes blandas: imágenes diagnósticas. Rev Chil Radiol 2007; 13: 109-121.

10. Enjorlas $O$. Classification and Management of the various superficial vascular anomalies: hemangioma and vascular malformation. J Dermatol 1997; 24: 701-710.

11. Omary R, Bettmann M, Cardella J, Bakal C, Schwartzberg $M$, Sacks $D$, et al. Quality improvement guidelines for the reporting and archiving of interventional radiology procedures. JVIR 2003; 4(9Pt2): S293-295.

12. Lee B, Do Y, Byun H, Choo I, Kim D, Huh S. Advanced Management of venous malformations with etanol sclerotherapy: mid-term results. J Vasc Surg 2003; 37: 533-538.

13. Rockman $C$, Rosen $R$, Jacobowitz $G$. Transcatheter embolization of extremity vascular malformations: the long-term success of multiple interventions. Ann Vasc Surg 2003; 17: 417-423. 